JOURNAL OF INTEGRAL EQUATIONS

AND APPLICATIONS

Volume 8, Number 4, Fall 1996

\title{
ON THE SOLUTION OF A SPECIAL KIND OF SINGULAR INTEGRAL EQUATION
}

\author{
OLAF HANSEN
}

ABSTRACT. An explicit inversion formula for Cauchy singular integral equations is used to numerically approximate the solution. We deal with the case where the solution is not continuously differentiable but is in a special class of Hölder continuous functions. We get results for the order of convergence in the supremum norm and for a weighted square norm.

1. Introduction. Let $L$ be a simple, closed smooth curve in $\mathbf{C}$ and $\psi \in C^{\alpha}(L, \mathbf{C}), \alpha \in(0,1)$. Here $C^{\alpha}(L, \mathbf{C})$ is the space of Hölder continuous functions on $L$ with the usual norm $\|\cdot\|_{C^{\alpha}(L, \mathbf{C})}$. The solution of the following singular integral equation

$$
\frac{1}{\pi i} \int_{L} \frac{\varphi(t)}{t-t_{0}} d t=\psi\left(t_{0}\right), \quad t_{0} \in L
$$

is given by

$$
\varphi\left(t_{0}\right)=\frac{1}{\pi i} \int_{L} \frac{\psi(t)}{t-t_{0}} d t, \quad t_{0} \in L,
$$

and we have $\varphi \in C^{\alpha}([0,2 \pi], \mathbf{C})[\mathbf{6}$, Section 27, formula (A)]. This means that we only need a suitable quadrature formula to find an approximation for $\varphi$.

A typical example for the application of the above formula (2) is the following:

Given a function $\psi \in C^{\alpha}([0,2 \pi], \mathbf{R})$, periodic, with

$$
\psi(0)=\psi(2 \pi)
$$

and

$$
\int_{0}^{2 \pi} \psi(x) d x=0
$$

\footnotetext{
Received by the editors on May 1, 1995, and in revised form on April 23, 1996.

AMS Mathematics Subject Classification. 45L10, 45E05, 65R20.

Key words and phrases. Integral equations, Hilbert transform, weakly singular solutions, graded meshes.

Copyright (C)1996 Rocky Mountain Mathematics Consortium
} 
then a solution $\varphi$ of the singular equation

$$
\frac{1}{2 \pi} \int_{0}^{2 \pi} \cot \left(\frac{x_{0}-x}{2}\right) \varphi(x) d x=\psi\left(x_{0}\right), \quad x_{0} \in[0,2 \pi]
$$

is given by

$$
\varphi\left(x_{0}\right)=-\frac{1}{2 \pi} \int_{0}^{2 \pi} \cot \left(\frac{x_{0}-x}{2}\right) \psi(x) d x, \quad x_{0} \in[0,2 \pi] .
$$

The function $\varphi$ is again an element of $C^{\alpha}([0,2 \pi], \mathbf{R})$ and is the only solution of (5) with

$$
\int_{0}^{2 \pi} \varphi(x) d x=0
$$

[6, formula (28.8)]. Again we see that it is only necessary to find an efficient quadrature formula for the right side of $(6)$ in order to get a good approximation for the function $\varphi$. If $\psi \in C^{2}([0,2 \pi], \mathbf{R})$ it is easy to find suitable quadrature formulas for $(6)$. In the sequel we treat the case where there exists one $x_{0} \in[0,2 \pi]$, where the derivative of $\psi$ does not exist $[\mathbf{1}]$.

The results of this paper are mainly of theoretical interest. In addition to the solution of the special equation (1), the studies are motivated by the following:

The solution of "Symm's integral equation"

$$
-\frac{1}{\pi} \int_{\Gamma} \log \left|t_{0}-t\right| u(t) d s(t)=f\left(t_{0}\right), \quad t_{0} \in \Gamma,
$$

where $\Gamma$ is a closed, simple, plane curve, leads to an equation of the form

$$
\begin{gathered}
-\frac{1}{\pi} \int_{0}^{2 \pi} \log \left|v(x)-v\left(x_{0}\right)\right|\left|v^{\prime}(x)\right| u(v(x)) d x=f\left(v\left(x_{0}\right)\right), \\
x_{0} \in[0,2 \pi] .
\end{gathered}
$$

Here $v:[0,2 \pi] \rightarrow \mathbf{R}^{2}$ is a parametrization of the curve $\Gamma$. If the curve is smooth with the exception of the point $v(\pi)$, it is known that the 
function $\left|v^{\prime}(x)\right| u(v(x))$ is smooth for $x \in[0,2 \pi] \backslash\{\pi\}$, if $f$ is smooth and $v$ is suitably chosen $[\mathbf{2}]$. This motivates the choice of the special function space $C_{\bullet}^{2, \alpha}([0,2 \pi], \mathbf{R})$, which we define in Section 2 . The analysis of (8) often considers the operator

$$
u \longrightarrow(K u)\left(x_{0}\right):=-\frac{1}{\pi} \int_{0}^{2 \pi} \log \left|v(x)-v\left(x_{0}\right)\right| u(x) d x
$$

as a perturbation of the operator

$$
u \longrightarrow(A u)\left(x_{0}\right):=-\frac{1}{\pi} \int_{0}^{2 \pi} \log \left(2 e^{-1 / 2}\left|\sin \left(\frac{x-x_{0}}{2}\right)\right|\right) u(x) d x .
$$

The inverse of $A$ is given by

$$
\begin{aligned}
\left(A^{-1} u\right)\left(x_{0}\right)= & -\frac{1}{2 \pi} \int_{0}^{2 \pi} \cot \left(\frac{x_{0}-x}{2}\right) u^{\prime}(x) d x \\
& +\frac{1}{2 \pi} \int_{0}^{2 \pi} u(x) d x, \quad u \in H_{\mathrm{per}}^{1}([0,2 \pi])
\end{aligned}
$$

[2]. Now we see that it may be of interest to calculate the Hilbert transform for functions which are smooth with the exception of one (or finitely many) point; see also the second example in Section 4. For smooth solutions, numerical methods for a wider class of singular integral equations can be found in $[\mathbf{3}]$ and $[4]$.

To approximate $\varphi$ in equation (6), we will interpolate $\psi$ with piecewise linear functions and construct a quadrature formula for these piecewise linear functions. The convergence of this method is based on the fact that the piecewise linear interpolants converge to $\psi$ in some Hölder norm.

In the next section we define the spaces of piecewise linear functions which we use and the quadrature formula. The third section contains the approximation results. In Section 4 some numerical examples demonstrate the results of Section 3.

2. Definitions and notations. The function space $C^{\alpha}([0,2 \pi], \mathbf{R})$, $\alpha \in(0,1)$, consists of those continuous functions $u$ on $[0,2 \pi]$ for which

$$
\sup _{s, t \in[0,2 \pi], t \neq s}\left|\frac{u(s)-u(t)}{|s-t|^{\alpha}}\right|<\infty
$$


holds. $C^{\alpha}([0,2 \pi], \mathbf{R})$ together with the norm

$$
\begin{aligned}
\|u\|_{C^{\alpha}([0,2 \pi], \mathbf{R})}:= & \sup _{s \in[0,2 \pi]}|u(s)| \\
& +\sup _{s, t \in[0,2 \pi], t \neq s}\left|\frac{u(s)-u(t)}{|s-t|^{\alpha}}\right|
\end{aligned}
$$

is a Banach space. The closed subspace $C_{\mathrm{per}}^{\alpha}([0,2 \pi], \mathbf{R})$ contains those functions $u \in C^{\alpha}([0,2 \pi], \mathbf{R})$ which fulfill

$$
u(0)=u(2 \pi) .
$$

For a function $\psi \in C_{\mathrm{per}}^{\alpha}([0,2 \pi], \mathbf{R}), \alpha \in(0,1)$, we define the Hilbert operator $\mathcal{H}$ by

$$
\begin{aligned}
&(\mathcal{H} \psi)\left(x_{0}\right)= \frac{1}{2 \pi} \int_{0}^{2 \pi} \cot \left(\frac{x_{0}-x}{2}\right) \psi(x) d x \\
&=\frac{1}{2 \pi} \int_{0}^{2 \pi} \cot \left(\frac{x_{0}-x}{2}\right)\left(\psi(x)-\psi\left(x_{0}\right)\right) d x \\
& \quad x_{0} \in[0,2 \pi] .
\end{aligned}
$$

The second equation follows from the fact that

$$
\int_{0}^{2 \pi} \cot \left(\frac{x_{0}-x}{2}\right) d x=0,
$$

where the principal value of the integral has to be taken. It is known $[5]$, that

$$
\mathcal{H} \in L\left(C_{\mathrm{per}}^{\alpha}([0,2 \pi], \mathbf{R}), C_{\mathrm{per}}^{\alpha}([0,2 \pi], \mathbf{R})\right), \quad \alpha \in(0,1),
$$

is a continuous linear operator, and that

$$
\|\mathcal{H}\|_{L\left(C_{\mathrm{per}}^{\alpha}([0,2 \pi], \mathbf{R}), C_{\mathrm{per}}^{\alpha}([0,2 \pi], \mathbf{R})\right)}=1 .
$$

We now define the function space, where we will approximate $\mathcal{H}$.

Definition 2.1. The function space $C_{\bullet}^{2, \alpha}([0,2 \pi], \mathbf{R}) \subset C_{\text {per }}^{\alpha}([0,2 \pi], \mathbf{R})$, $\alpha \in(0,1)$, is defined as the set of all such $\psi$ for which 
(a) $\psi \in C_{\text {per }}^{\alpha}([0,2 \pi], \mathbf{R}) \cap C^{2}([0, \pi)) \cap C^{2}((\pi, 2 \pi])$.

(b) There exists a $C_{0}=C_{0}(\psi)$ :

$$
\left|\psi^{(2)}(x)\right| \leq C_{0}|x-\pi|^{\alpha-2}, \quad x \in[0,2 \pi] .
$$

Remark. We will always assume that the constant $C_{0}(u)$ also fulfills

$$
\begin{aligned}
|\psi(x)| & \leq C_{0}, \\
\left|\psi^{(1)}(x)\right| & \leq C_{0}|x-\pi|^{\alpha-1}, \quad \\
|\psi(x)-\psi(s)| & \leq C_{0}|x-s|^{\alpha}, \quad x \in[0,2 \pi],
\end{aligned}
$$

In order to approximate functions in $C_{\bullet}^{2, \alpha}([0,2 \pi], \mathbf{R})$, we use piecewise linear functions, which we define now.

Definition 2.2. For $n \in \mathbf{N}, n \geq 2$ and $q \geq 1$, we define a partition of $[0,2 \pi]$

$$
0=x_{0}^{(n, q)}<x_{1}^{(n, q)}<\cdots<x_{n}^{(n, q)}=2 \pi
$$

by

$$
x_{j}^{(n, q)}:= \begin{cases}\pi\left[1-(1-2 j / n)^{q}\right] & j=0(1)[n / 2], \\ \pi\left[1+(1-2(n-j) / n)^{q}\right] & j=[n / 2]+1,(1) n,\end{cases}
$$

the step width

$$
h_{j}^{(n, q)}:=x_{j}^{(n, q)}-x_{j-1}^{(n, q)}, \quad j=1(1) n,
$$

and the weights for the trapezoidal rule

$$
\begin{aligned}
\omega_{0}^{(n, q)} & :=\frac{h_{1}^{(n, q)}+h_{n}^{(n, q)}}{2} \\
\omega_{j}^{(n, q)} & :=\frac{h_{j}^{(n, q)}+h_{j+1}^{(n, q)}}{2}, \quad j=1(1) n-1 . \\
\omega_{n}^{(n, q)} & :=\frac{h_{n}^{(n, q)}+h_{1}^{(n, q)}}{2}
\end{aligned}
$$


The function space $\mathcal{P}^{(n, q)} \subset C_{\text {per }}^{\alpha}([0,2 \pi], \mathbf{R}), \alpha \in(0,1)$, of continuous, piecewise linear functions is defined by

$$
\mathcal{P}^{(n, q)}:=\left\{\psi \in C_{\mathrm{per}}^{\alpha}([0,2 \pi], \mathbf{R})|\psi|_{\left[x_{j}^{(n, q)}, x_{j+1}^{(n, q)}\right]} \text { linear, } j=0(1) n-1\right\} .
$$

For $\psi \in C_{\bullet}^{2, \alpha}([0,2 \pi], \mathbf{R})$ the interpolant $P^{(n, q)} \psi \in \mathcal{P}^{(n, q)}$ is given in the usual manner by

$$
\left(P^{(n, q)} \psi\right)\left(x_{j}^{(n, q)}\right)=\psi\left(x_{j}^{(n, q)}\right), \quad j=0(1) n .
$$

Furthermore, we define two norms on $\mathbf{R}^{n}$ :

$$
\begin{aligned}
& \left\|\left(u_{j}\right)_{j}\right\|_{2, n, q}:=\left(\sum_{j=0}^{n-1} \omega_{j}^{(n, q)} u_{j}^{2}\right)^{1 / 2} \\
& \left\|\left(u_{j}\right)_{j}\right\|_{\infty, n}:=\max _{j=0}^{n-1}\left|u_{j}\right| \\
& \left(u_{j}\right)_{j}=\left(u_{0}, u_{1}, \ldots, u_{n-1}\right)^{T} \in \mathbf{R}^{n} .
\end{aligned}
$$

Remark. We have

$$
\max _{j=1(1) n}\left\{h_{j}^{(n, q)}\right\}=\mathcal{O}_{n \rightarrow \infty}(1 / n)
$$

for a given $q \geq 1$.

To treat the singularity of the function $\cot (\cdot)$, we define an auxiliary function:

$$
S(x):=\frac{1}{x}+\frac{1}{x-\pi}+\frac{1}{x+\pi}, \quad x \in \mathbf{R} \backslash\{-\pi, 0, \pi\} .
$$

We get

$$
R(x):=\cot (x)-S(x) \in C^{\infty}\left(\left[-\frac{3}{2} \pi, \frac{3}{2} \pi\right]\right)
$$


if we define $R(0):=R(\pi):=R(-\pi):=0$. Then our approximation $\hat{\mathcal{H}}^{(n, q)}$ for $\mathcal{H}$ on $\mathcal{P}^{(n, q)}$ is defined by

$$
\begin{aligned}
\left(\hat{\mathcal{H}}^{(n, q)} u\right)(s):= & \frac{1}{2 \pi} \int_{0}^{2 \pi} P^{(n, q)}\left(R\left(\frac{s-\cdot}{2}\right)(u(\cdot)-u(s))\right)(x) d x \\
& +\frac{1}{2 \pi} \int_{0}^{2 \pi} S\left(\frac{s-x}{2}\right)(u(x)-u(s)) d x,
\end{aligned}
$$

$u \in \mathcal{P}^{(n, q)}$. All integrals on the righthand side of (27) can be calculated explicitly, and the first term on the right side gives the trapezoidal rule for the integral

$$
\frac{1}{2 \pi} \int_{0}^{2 \pi} R\left(\frac{s-x}{2}\right)(u(x)-u(s)) d x .
$$

Finally we define the approximation $\mathcal{H}^{(n, q)}$ for $\mathcal{H}$ by

$$
\mathcal{H}^{(n, q)}:=P^{(n, q)} \circ \hat{H}^{(n, q)} \circ P^{(n, q)} .
$$

3. Results. The first lemma describes the approximation properties of piecewise linear interpolants in our Hölder spaces.

Lemma 3.1. Let $u \in C_{\bullet}^{2, \alpha}([0,2 \pi], \mathbf{R})$. For $\alpha^{\prime} \in(0, \alpha)$ and $q \geq 1$, there exists a constant $C_{1}=C_{1}\left(C_{0}(u), q, \alpha, \alpha^{\prime}\right)$ (see Definition 2.1) such that

$$
\left\|u-P^{(n, q)} u\right\|_{C^{\alpha^{\prime}}([0,2 \pi], \mathbf{R})} \leq C_{1}\left\{\begin{array}{ll}
1 / n^{q\left(\alpha-\alpha^{\prime}\right)} & q \leq\left(2-\alpha^{\prime}\right) /\left(\alpha-\alpha^{\prime}\right) \\
1 / n^{2-\alpha^{\prime}} & q \geq\left(2-\alpha^{\prime}\right) /\left(\alpha-\alpha^{\prime}\right)
\end{array} .\right.
$$

Proof. It is known that there exists a $\tilde{C}$ with

$$
\left\|u-P^{(n, q)} u\right\|_{C^{0}([0,2 \pi], \mathbf{R})} \leq \tilde{C}\left\{\begin{array}{ll}
1 / n^{q \alpha} & q \alpha<2 \\
1 / n^{2} & q \alpha \geq 2
\end{array} .\right.
$$

See [7]. We consider only the case $n$ even. It is sufficient to prove

$$
\begin{array}{r}
\sup _{x, s \in[0,2 \pi], x \neq s}\left|\frac{\left(P^{(n, q)} u-u\right)(x)-\left(P^{(n, q)} u-u\right)(s)}{|x-s|^{\alpha^{\prime}}}\right| \\
\leq \gamma_{1} \begin{cases}1 / n^{q\left(\alpha-\alpha^{\prime}\right)} & q<\left(2-\alpha^{\prime}\right) /\left(\alpha-\alpha^{\prime}\right), \\
1 / n^{2-\alpha^{\prime}} & q \geq\left(2-\alpha^{\prime}\right) /\left(\alpha-\alpha^{\prime}\right),\end{cases}
\end{array}
$$


where $\gamma_{1}=\gamma_{1}\left(C_{0}(u)\right)$ is a constant not depending on $n$.

We first consider the case $x, s \geq \pi, x, s \in\left[x_{j}^{(n, q)}, x_{j+1}^{(n, q)}\right], j \in$ $\{n / 2, \ldots, n-1\}$. In the sequel we will omit the upper index $(n, q)$.

The following two cases are treated separately:

(a) $x, s \in\left[x_{n / 2}, x_{n / 2+1}\right]=\left[\pi, x_{n / 2+1}\right]$.

(b) $x, s \in\left[x_{n / 2+\tau}, x_{n / 2+\tau+1}\right], \tau \in\{1, \ldots, n / 2-1\}$.

Case a. Here we have $h:=h_{n / 2+1}=\pi 2^{q} / n^{q}$. We get

$$
\begin{aligned}
\mid(P u-u)(x)- & (P u-u)(s) \mid \\
= & \mid \frac{x-\pi}{h} u\left(x_{n / 2+1}\right)+\frac{x_{n / 2+1}-x}{h} u\left(x_{n / 2}\right)-u(x) \\
& -\left(\frac{s-\pi}{h} u\left(x_{n / 2+1}\right)+\frac{x_{n / 2+1}-s}{h} u\left(x_{n / 2}\right)-u(s)\right) \mid \\
= & \left|\frac{x-s}{h} u\left(x_{n / 2+1}\right)+\frac{s-x}{h} u\left(x_{n / 2}\right)+u(s)-u(x)\right| \\
\leq & \frac{|x-s|}{h}\left|u\left(x_{n / 2+1}\right)-u\left(x_{n / 2}\right)\right|+|u(x)-u(s)| \\
\leq & C_{0}(u)|x-s| h^{\alpha-1}+C_{0}(u)|x-s|^{\alpha} .
\end{aligned}
$$

This implies

$$
\begin{aligned}
\frac{|(P u-u)(x)-(P u-u)(s)|}{|x-s|^{\alpha^{\prime}}} & \leq 2 C_{0} h^{\alpha-\alpha^{\prime}} \\
& =2 C_{0} \pi^{\alpha-\alpha^{\prime}} 2^{q\left(\alpha-\alpha^{\prime}\right)} \frac{1}{n^{q\left(\alpha-\alpha^{\prime}\right)}} \\
& =\gamma_{2} \frac{1}{n^{q\left(\alpha-\alpha^{\prime}\right)}}
\end{aligned}
$$

where $\gamma_{2}=2 C_{0} \pi^{\alpha-\alpha^{\prime}} 2^{q\left(\alpha-\alpha^{\prime}\right)}$.

Case b. Now we have

$$
x_{n / 2+\tau}-\pi=\pi 2^{q} \frac{\tau^{q}}{n^{q}}
$$

and we define

$$
h:=h_{n / 2+\tau+1} \leq \pi q 2^{q} \frac{(1+\tau)^{q-1}}{n^{q}} .
$$


Using (14) an elementary calculation gives

$$
|(P u-u)(x)-(P u-u)(s)| \leq C_{0}(u)|x-s| h\left(x_{n / 2+\tau}-\pi\right)^{\alpha-2},
$$

which shows

$$
\begin{aligned}
& \frac{|(P u-u)(x)-(P u-u)(s)|}{|x-s|^{\alpha^{\prime}}} \\
& \leq C_{0}(u) h^{2-\alpha^{\prime}}\left(x_{n / 2+\tau}-\pi\right)^{\alpha-2} \\
& \leq C_{0} \pi^{\alpha-\alpha^{\prime}} 2^{q\left(\alpha-\alpha^{\prime}\right)} q^{2-\alpha^{\prime}}\left(\frac{1+\tau}{\tau}\right)^{(q-1)\left(2-\alpha^{\prime}\right)} \\
& \quad \cdot \frac{1}{n^{q\left(\alpha-\alpha^{\prime}\right)} \tau^{q\left(\alpha-\alpha^{\prime}\right)+\alpha^{\prime}-2}} \\
& \leq \gamma_{3} \begin{cases}1 / n^{q\left(\alpha-\alpha^{\prime}\right)} & q<\left(2-\alpha^{\prime}\right) /\left(\alpha-\alpha^{\prime}\right), \\
1 / n^{2-\alpha^{\prime}} & q \geq\left(2-\alpha^{\prime}\right) /\left(\alpha-\alpha^{\prime}\right)\end{cases}
\end{aligned}
$$

where $\gamma_{3}:=C_{0}(u) \pi^{\alpha-\alpha^{\prime}} 2^{q\left(\alpha-\alpha^{\prime}\right)} q^{2-\alpha^{\prime}} \max _{\tau \in \mathbf{N}}\left\{((1+\tau) / \tau)^{(q-1)\left(2-\alpha^{\prime}\right)}\right\}$. Now the cases a) and b) show together with the symmetry of the problem that inequality (32) is fulfilled for $x, s \in\left[x_{j}, x_{j+1}\right], j \in$ $\{0, \ldots, n-1\}$ and $\gamma_{1}:=\max \left\{\gamma_{2}, \gamma_{3}\right\}$.

Let $x, s \in[0,2 \pi]$ be contained in different intervals $\left[x_{j}, x_{j+1}\right]$. We assume $x<s$ and

$$
x \in\left[x_{j_{1}}, x_{j_{1}+1}\right], \quad s \in\left[x_{j_{2}}, x_{j_{2}+1}\right] .
$$

Then we get

$$
\begin{aligned}
& \frac{\mid(P u-u)(x)-}{\left.|x-s|^{\alpha^{\prime}}-u\right)(s) \mid} \\
& \leq \frac{\left|(P u-u)(x)-(P u-u)\left(x_{j_{1}+1}\right)\right|}{|x-s|^{\alpha^{\prime}}} \\
&+\frac{\left|(P u-u)(s)-(P u-u)\left(x_{j_{2}}\right)\right|}{|x-s|^{\alpha^{\prime}}} \\
& \leq \frac{\left|(P u-u)(x)-(P u-u)\left(x_{j_{1}+1}\right)\right|}{\left|x-x_{j_{1}+1}\right|^{\alpha^{\prime}}} \\
&+\frac{\left|(P u-u)(x)-(P u-u)\left(x_{j_{2}}\right)\right|}{\left|x-x_{j_{2}}\right|^{\alpha^{\prime}}} \\
& \leq 2 \max \left\{\gamma_{2}, \gamma_{3}\right\} \begin{cases}1 / n^{q\left(\alpha-\alpha^{\prime}\right)} & q<\left(2-\alpha^{\prime}\right) /\left(\alpha-\alpha^{\prime}\right) \\
1 / n^{2-\alpha^{\prime}} & q \geq\left(2-\alpha^{\prime}\right) /\left(\alpha-\alpha^{\prime}\right)\end{cases}
\end{aligned}
$$


by inequality (32). Here the facts

$$
(P u-u)\left(x_{j_{1}+1}\right)=(P u-u)\left(x_{j_{2}}\right)=0,
$$

and

$$
\left|\frac{x-x_{j_{1}+1}}{x-s}\right|^{\alpha^{\prime}},\left|\frac{x-x_{j_{2}}}{x-s}\right|^{\alpha^{\prime}} \leq 1
$$

have been used. This proves (32) with $\gamma_{1}=2 \max \left\{\gamma_{2}, \gamma_{3}\right\}$.

The next lemma shows that the quadrature formula in equation (27) is sufficiently exact for our purpose.

Lemma 3.2. Let $f \in C^{2}([0,2 \pi], \mathbf{R})$ and $u \in C_{\bullet}^{2, \alpha}([0,2 \pi], \mathbf{R})$. Then we have

$$
\left.\left|\int_{0}^{2 \pi} f(x)\left(P^{(n, q)} u\right)(x)-P^{(n, q)}\left(f \cdot\left(P^{(n, q)} u\right)\right)(x) d x\right| \leq C_{2} / n^{2}\right)
$$

where $C_{2}=C_{2}\left(C_{0}(u), q,\left\|f^{\prime}\right\|_{C^{0}},\left\|f^{\prime \prime}\right\|_{C^{0}}\right)$.

Proof. In the following we will omit the upper index $(n, q)$. We have

$$
\begin{aligned}
F_{n}: & =\int_{0}^{2 \pi}|f(x) P u(x)-P(f \cdot P u)(x)| d x \\
& =\sum_{j=0}^{n-1} \int_{x_{j}}^{x_{j+1}}\left|\Delta_{j}(x)\right| d x,
\end{aligned}
$$

where

$(34)$

$$
\begin{aligned}
\Delta_{j}(x):= & f(x)\left(\frac{x-x_{j}}{h_{j+1}} u\left(x_{j+1}\right)+\frac{x_{j+1}-x}{h_{j+1}} u\left(x_{j}\right)\right) \\
& -\left(\frac{x-x_{j}}{h_{j+1}} u\left(x_{j+1}\right) f\left(x_{j+1}\right)+\frac{x_{j+1}-x}{h_{j+1}} u\left(x_{j}\right) f\left(x_{j}\right)\right) \\
= & \frac{\left(x-x_{j}\right)\left(x_{j+1}-x\right)}{h_{j+1}}\left(u\left(x_{j+1}\right) h_{j+1} f\left[x_{j}, x, x_{j+1}\right]\right. \\
& \left.+f\left[x_{j}, x\right]\left(u\left(x_{j+1}\right)-u\left(x_{j}\right)\right)\right) .
\end{aligned}
$$


If $\pi \notin\left[x_{j}, x_{j+1}\right]$, we further have

(36)

$\Delta_{j}(x)=\left(x-x_{j}\right)\left(x_{j+1}-x\right)\left(u\left(x_{j+1}\right) f\left[x_{j}, x, x_{j+1}\right]+f\left[x_{j}, x\right] u\left[x_{j}, x_{j+1}\right]\right)$.

Now we consider only the case $n$ even.

(a) $j=n / 2$. Then it is easy to see, with the help of (35) and (17), that there exists a constant $\gamma_{1}=\gamma_{1}\left(C_{0}(u),\left\|f^{\prime}\right\|_{C^{0}([0,2 \pi])},\left\|f^{\prime \prime}\right\|_{C^{0}([0,2 \pi])}\right)$ for which

$$
\left|\Delta_{j}(x)\right| \leq \gamma_{1} \frac{1}{n^{q(\alpha+1)}}
$$

(b) $j=n / 2+\tau, \tau \in\{1, \ldots, n / 2-1\}$. Then (36) and a simple calculation show the existence of a constant $\gamma_{2}=\gamma_{2}\left(C_{0}(u),\left\|f^{\prime}\right\|_{C^{0}([0,2 \pi])}\right.$, $\left.\left\|f^{\prime \prime}\right\|_{C^{0}([0,2 \pi])}\right)$ with

$$
|| \Delta_{j}(x) \mid \leq \gamma_{2}\left(\frac{(1+\tau)^{2(q-1)}}{n^{2 q}}+\frac{(1+\tau)^{q \alpha+q-2}}{n^{q(\alpha+1)}}\right) .
$$

Here

$$
h_{j+1} \leq \operatorname{cst} . \frac{(1+\tau)^{q-1}}{n^{q}}
$$

and (14) have been used. This gives

$$
\begin{aligned}
F_{n} \leq & 2 h_{n / 2+1} \gamma_{1} \frac{1}{n^{q(\alpha+1)}} \\
& +2 \gamma_{2} \sum_{\tau=1}^{n / 2-1} h_{n / 2+\tau+1}\left(\frac{(1+\tau)^{2(q-1)}}{n^{2 q}}+\frac{(1+\tau)^{q \alpha+q-2}}{n^{q(\alpha+1)}}\right)
\end{aligned}
$$

which shows together with (39) the existence of a constant $\gamma_{3}=$ $\gamma_{3}\left(\gamma_{2}, \gamma_{1}\right)$ with

$$
\begin{aligned}
F_{n} \leq \gamma_{3}\left(\frac{1}{n^{q(\alpha+2)}}+\frac{1}{n^{3 q}} \sum_{\tau=1}^{n / 2-1}\right. & (1+\tau)^{3(q-1)} \\
& \left.\quad+\frac{1}{n^{q(\alpha+2)}} \sum_{\tau=1}^{n / 2-1}(1+\tau)^{q \alpha+2 q-3}\right) .
\end{aligned}
$$


Because of $q \geq 1$, we have

$$
\sum_{\tau=1}^{n / 2-1}(1+\tau)^{3(q-1)} \leq \mathrm{cst} \cdot n^{3 q-2},
$$

and

$$
\sum_{\tau=1}^{n / 2-1}(1+\tau)^{q(\alpha+2)-3} \leq \text { cst. } n^{q \alpha+2 q-2} .
$$

Together with (40), this implies

$$
F_{n} \leq C_{2}\left(\gamma_{3}\right) \frac{1}{n^{2}}
$$

Thus the lemma is proved.

Corollary 3.3. Let $u \in C_{\bullet}^{2, \alpha}([0,2 \pi], \mathbf{R})$. Then there exists a constant $C_{3}\left(C_{0}(u), \alpha, q\right)$ for which we have

$$
\left.\mid \mathcal{H}-\mathcal{H}^{(n, q)}\right)\left(P^{(n, q)} u\right)(s) \mid \leq C_{3} \frac{1}{n^{2}}, \quad j \in\{0, \ldots, n-1\}, \quad n \in \mathbf{N} .
$$

Proof. The statement follows from Lemma 3.2 with

$$
f_{s}(x):=R\left(\frac{s-\cdot}{2}\right)
$$

and

$$
v_{s}(x):=u(x)-u(s)
$$

instead of $u(x)$. We have $C_{0}\left(v_{s}\right) \leq 2 C_{0}(u)$ for all $s \in[0,2 \pi]$, and $(26)$ implies

$$
\max _{s \in[0,2 \pi]}\left\{\left\|f_{s}^{\prime}\right\|_{C^{0}},\left\|f_{s}^{\prime \prime}\right\|_{C^{0}}\right\}<\infty
$$

Theorem 3.4. Let $u \in C_{\bullet}^{2, \alpha}([0,2 \pi], \mathbf{R}), \alpha \in(0,1)$ be given. For $\alpha^{\prime} \in(0, \alpha)$, there exists a constant $C_{4}=C_{4}\left(C_{0}(u), q, \alpha, \alpha^{\prime}\right)$ for which

(41) $\left\|\left((\mathcal{H} u)\left(x_{j}^{(n, q)}\right)-\left(\mathcal{H}^{(n, q)} u\right)\left(x_{j}^{(n, q)}\right)\right)_{j}\right\|_{\infty, n}$

$$
\leq C_{4}\left\{\begin{array}{ll}
1 / n^{q\left(\alpha-\alpha^{\prime}\right)} & q \leq\left(2-\alpha^{\prime}\right) /\left(\alpha-\alpha^{\prime}\right) \\
1 / n^{2-\alpha^{\prime}} & q \geq\left(2-\alpha^{\prime}\right) /\left(\alpha-\alpha^{\prime}\right)
\end{array} .\right.
$$


Remark. The number $\alpha^{\prime}$ does not appear on the left side of equation (41) but only on the righthand side (for the reason, see the following proof). This means that for $q$ big enough we get convergence for every grid point faster than $\mathcal{O}_{n \rightarrow \infty}\left(1 / n^{\beta}\right)$ for every $\beta \in(1,2)$.

Proof. By Corollary 3.3 we have

$$
\begin{aligned}
\mid(\mathcal{H} u)\left(x_{j}^{(n, q)}\right)- & \left(\mathcal{H}^{(n, q)} u\right)\left(x_{j}^{(n, q)}\right) \mid \\
\leq & \left|\left(\mathcal{H}\left(u-\mathcal{P}^{(n, q)} u\right)\right)\left(x_{j}^{(n, q)}\right)\right| \\
& +\left|\left(\left(\mathcal{H}-\mathcal{H}^{(n, q)}\right)\left(\mathcal{P}^{(n, q)} u\right)\right)\left(x_{j}^{(n, q)}\right)\right| \\
\leq & \|H\|_{L\left(C^{\alpha^{\prime}}([0,2 \pi], \mathbf{R})\right)} \mid u-\mathcal{P}^{(n, q)} u \|_{C^{\alpha^{\prime}}([0,2 \pi], \mathbf{R})} \\
& +C_{3} \frac{1}{n^{2}} .
\end{aligned}
$$

The fact that $\|\mathcal{H}\|_{L\left(C^{\alpha^{\prime}}([0,2 \pi], \mathbf{R}), C^{\left.\alpha^{\prime}([0,2 \pi], \mathbf{R})\right)}\right.}=1$ and Lemma 3.1 prove the result.

To study the error with respect to the $\|\cdot\|_{2, n, q}$-norm, we have to take a closer look at $\mathcal{H}\left(u-P^{(n, q)} u\right)$.

Lemma 3.5. Let $u \in C_{\bullet}^{2, \alpha}([0,2 \pi], \mathbf{R})$. Then there exists a constant $C_{5}=C_{5}\left(C_{0}(u), \alpha, q\right)$ which fulfills

$$
\begin{aligned}
& \left\|\left(\left((\mathcal{H} u)-\left(\mathcal{H} P^{(n, q)} u\right)\right)\left(x_{j}^{(n, q)}\right)\right)_{j}\right\|_{2, n, q} \\
& \leq C_{5} \begin{cases}\ln (n) / n^{q(\alpha+1 / 2)} & q \in[1,4 /(2 \alpha+1)), \\
\ln (n)^{3 / 2} / n^{2} & q=4 /(2 \alpha+1), \\
\ln (n) / n^{2} & q \in(4 /(2 \alpha+1), 2 / \alpha], \\
1 / n^{\beta} & q>2 / \alpha, \beta \in(1,2) \text { arbitrary. }\end{cases}
\end{aligned}
$$

Proof. The inequality for $q>2 / \alpha$ is a consequence of Theorem 3.4. We will only consider the case $n$ even, and we assume first that we have proved the following inequality

$$
\begin{aligned}
& \left|\left(\mathcal{H} u-\mathcal{H} P^{(n, q)} u\right)\left(x_{n / 2+\tau}\right)\right| \\
& \quad \leq \frac{\gamma_{0}}{n^{q \alpha}} \ln (n) \begin{cases}\tau^{-q} & q \in[1,2 /(1+\alpha)] \\
\tau^{q \alpha-2} & q \in(2 /(1+\alpha), 2 / \alpha]\end{cases}
\end{aligned}
$$


$\tau \in\{1, \ldots, n / 2-1\}, \gamma_{0}=\gamma_{0}\left(C_{0}(u), \alpha, q\right)$.

We first deal with the case $q \in[1,2 /(1+\alpha)]$. Because of symmetry, we get

(45) $\quad\left\|\left(\mathcal{H} u\left(x_{j}\right)-\mathcal{H}^{(n, q)} u\left(x_{j}\right)\right)_{j}\right\|_{2, n, q}^{2}$

$$
\leq \gamma_{1}\left(\sum_{\tau=1}^{n / 2-1} \frac{(1+\tau)^{q-1}}{n^{q}}\left(\frac{\ln (n) \tau^{-q}}{n^{q \alpha}}\right)^{2}+\frac{1}{n^{q}} \frac{1}{n^{2 q \alpha}}\right)
$$

with a constant $\gamma_{1}>0$. Here

$$
\omega_{n / 2+\tau} \leq \operatorname{cst} . \frac{(1+\tau)^{q-1}}{n^{q}}
$$

and

$$
\omega_{n / 2} \leq 1 / n^{q}
$$

have been used. Now (45) gives

$$
\begin{aligned}
\left\|\left(\mathcal{H} u\left(x_{j}\right)-\mathcal{H}^{(n, q)} u\left(x_{j}\right)\right)_{j}\right\|_{2, n, q}^{2} & \leq \gamma_{2}\left(\gamma_{1}\right) \frac{\ln (n)^{2}}{n^{q(1+2 \alpha)}} \sum_{\tau=1}^{n / 2-1}(1+\tau)^{-1-q} \\
& \leq \gamma_{3} \frac{\ln (n)^{2}}{n^{q(2 \alpha+1)}}
\end{aligned}
$$

with

$$
\gamma_{3}:=\gamma_{2} \sum_{\tau=1}^{\infty}(1+\tau)^{-q-1}<\infty, \quad \text { because } q \geq 1 .
$$

This proves $(43)$ in the case $q \in[1,2 /(1+\alpha)] \subset[1,4 /(2 \alpha+1))$.

If $q \in(2 /(1+\alpha), 2 / \alpha]$ we get

$$
\begin{aligned}
\|\left(\mathcal{H} u\left(x_{j}\right)\right. & \left.-\mathcal{H}^{(n, q)} u\left(x_{j}\right)\right)_{j} \|_{2, n, q}^{2} \\
& \leq \gamma_{4}\left(\sum_{\tau=1}^{n / 2-1} \frac{(1+\tau)^{q-1}}{n^{q}}\left(\frac{\ln (n) \tau^{q \alpha-2}}{n^{q \alpha}}\right)^{2}+\frac{1}{n^{q}} \frac{1}{n^{2 q \alpha}}\right) \\
& \leq \gamma_{5} \frac{\ln (n)^{2}}{n^{q(2 \alpha+1)}} \sum_{\tau=1}^{n / 2-1}(1+\tau)^{2 q \alpha+q-5}
\end{aligned}
$$


$\gamma_{4}, \gamma_{5}>0$ independent of $n$.

The following three cases have to be considered:

(a) $2 q \alpha+q-5=-1 \Leftrightarrow q=4 /(2 \alpha+1)>2 /(1+\alpha)$. Then we have

$$
\begin{aligned}
\sum_{\tau=1}^{n / 2-1}(1+\tau)^{q \alpha+q-5} & =\sum_{\tau=1}^{n / 2-1} \frac{1}{1+\tau} \\
& \leq \operatorname{cst} . \ln (n)
\end{aligned}
$$

(b) $2 q \alpha+q-5<-1 \Rightarrow q \in(2 /(1+\alpha), 4 /(2 \alpha+1))$. Here we have

$$
\sum_{\tau=1}^{n / 2-1}(1+\tau)^{2 q \alpha+q-5} \leq \sum_{\tau=1}^{\infty}(1+\tau)^{2 q \alpha+q-5}<\infty
$$

(c) $2 q \alpha+q-5>-1 \Rightarrow q \in(4 /(2 \alpha+1), 2 / \alpha]$. Then we have

$$
\sum_{\tau=1}^{n / 2-1}(1+\tau)^{2 q \alpha+q-5} \leq \text { cst. } n^{2 q \alpha+q-4} .
$$

(46) and the three inequalities show (43) for $q \in(2 /(1+\alpha), 2 / \alpha]$.

Now we want to sketch the proof of (44) for $j=n / 2+\tau, \tau \in$ $\{1, \ldots, n / 2-1\}$. We have

$$
\mathcal{H}(u-P u)\left(x_{n / 2+\tau}\right)=\frac{1}{2 \pi} \sum_{k=0}^{n-1} I_{n / 2+\tau, k}
$$

where

$$
I_{n / 2+\tau, k}:=\int_{x_{k}}^{x_{k+1}} \cot \left(\frac{x_{n / 2+\tau}-s}{2}\right)(u(s)-P u(s)) d s .
$$

In (47) the sum

$$
\sum_{k=0}^{n / 2-1}\left|I_{n / 2+\tau, k}\right|
$$


can be estimated by

$$
\text { cst. } \sum_{k=n / 2}^{n-1}\left|I_{n / 2+\tau, k}\right|
$$

because the singularity of $\cot \left(\left(x_{n / 2+\tau}-\cdot\right) / 2\right)$ lies between $\pi$ and $2 \pi$. The sum in (48) is split into

$$
\left|I_{n / 2+\tau, n / 2}\right|+\sum_{k=n / 2+1}^{n / 2+\tau-1}\left|I_{n / 2+\tau, k}\right|+\sum_{k=n / 2+\tau}^{n-1}\left|I_{n / 2+\tau, k}\right|
$$

and we will treat only the second summand.

By equation (14) and an estimation for the cotangents we get a constant $\gamma_{6}$ for which

$$
\begin{aligned}
& \left|I_{n / 2+\tau, k}\right| \leq \gamma_{6}\left(x_{k}-\pi\right)^{\alpha-2} \\
& \cdot \int_{x_{k}}^{x_{k+1}}\left(\frac{1}{x_{n / 2+\tau}-s}\left(s-x_{k}\right)\left(x_{k+1}-s\right)\right) d s
\end{aligned}
$$

$k \in\{n / 2+1, \ldots, n / 2+\tau-1\}$ holds. The integral on the righthand side of equation (50) is estimated by

$$
\frac{\Gamma(1)^{2}}{\Gamma(4)} \frac{1}{x_{n / 2+\tau}-x_{k}}{ }_{2} F_{1}\left(1,2,4, \frac{h_{k+1}}{x_{n / 2+\tau}-x_{k}}\right) h_{k+1}^{3}
$$

(see $\left[8\right.$, pp. 1, 20]) which is bounded by cst. $h_{k+1}^{2}$ for all $k<n / 2+\tau-1$. The definition (18) of the $x_{j}$ implies, therefore,

$$
\left|I_{n / 2+\tau, k}\right| \leq \gamma_{7} \frac{1}{n^{q \alpha}}(1+\kappa)^{q(\alpha+1)-3} \frac{1}{\tau^{q}-\kappa^{q}},
$$

with a constant $\gamma_{7}>0$ and $k=n / 2+\kappa, \kappa \in\{1, \ldots, \tau-1\}$. In the 
next step we estimate

$(52)$

$$
\begin{aligned}
\sum_{\kappa=1}^{\tau-1} \frac{\kappa^{q(\alpha+1)-3}}{\tau^{q}-\kappa^{q}}=\tau^{q \alpha-2}\left(\sum_{\kappa=1}^{[(r-1) / 2]} \frac{1}{\tau}\left(\frac{\kappa}{\tau}\right)^{q(\alpha+1)-3} \frac{1}{1-(\kappa / \tau)^{q}}\right. \\
\left.+\sum_{\kappa=[(\tau-1) / 2]+1}^{\tau-1} \frac{1}{\tau}\left(\frac{\kappa}{\tau}\right)^{q(\alpha+1)-3} \frac{1}{1-(\kappa / \tau)^{q}}\right) \\
\leq 4 \tau^{q \alpha-2}\left(\sum_{\kappa=1}^{[(\tau-1) / 2]} \frac{1}{\tau}\left(\frac{\kappa}{\tau}\right)^{q(\alpha+1)-3}\right. \\
\left.+\sum_{\kappa=[(\tau-1) / 2]+1}^{\tau-1} \frac{1}{\tau} \frac{1}{1-(\kappa / \tau)^{q}}\right) .
\end{aligned}
$$

The first term on the righthand side can be estimated directly or with the help of the integral

$$
\int_{1 /(2 \tau)}^{1 / 2} x^{q(\alpha+1)-3} d x
$$

We get

$$
\sum_{\kappa=1}^{[(\tau-1) / 2]} \frac{1}{\tau}\left(\frac{\kappa}{\tau}\right)^{q(\alpha+1)-3} \leq \gamma_{8} \begin{cases}\tau^{2-q(\alpha+1)} & q \in[1,2 /(1+\alpha)) \\ (1+\ln (\tau)) & q=2 /(1+\alpha) \\ 1 & q \in(2 /(1+\alpha), 2 / \alpha]\end{cases}
$$

Because of the convexity of the function $1 /\left(1-x^{q}\right), x \in[0,1)$, we can estimate the second sum in (52) by

$$
\int_{1 / 2}^{1-1 /(2 \tau)} \frac{1}{1-x^{q}} d x
$$

This gives

$$
\begin{aligned}
\sum_{\kappa=[(\tau-1) / 2]+1}^{\tau-1} \frac{1}{\tau} \frac{1}{1-(\kappa / \tau)^{q}} & \leq \gamma_{9}(1+\ln (\tau)) \\
& \leq \gamma_{9}(1+\ln (n))
\end{aligned}
$$


Now (53) and (54) together with (52) give

$$
\sum_{\kappa=1}^{\tau-1} \frac{\kappa^{q(\alpha+1)-3}}{\tau^{q}-\kappa^{q}} \leq \frac{\gamma_{10} \ln (n)}{n^{q \alpha}} \begin{cases}\tau^{q \alpha-2} & q \in[2 /(1+\alpha), 2 / \alpha] \\ \tau^{-q} & q \in[1,2 /(1+\alpha)]\end{cases}
$$

The other two terms in (49) can be estimated in a similar way. This proves (44) and our lemma.

Theorem 3.6. Let $u \in C_{\bullet}^{2, \alpha}([0,2 \pi], \mathbf{R})$. There exists a constant $C_{6}\left(C_{0}(u), \alpha, q\right)$ for which

$$
\begin{aligned}
& \left\|\left(\mathcal{H} u\left(x_{j}^{(n, q)}\right)-\mathcal{H}^{(n, q)} u\left(x_{j}^{(n, q)}\right)\right)_{j}\right\|_{2, n, q} \\
& \leq C_{6} \begin{cases}\ln (n) / n^{q(\alpha+1 / 2)} & q \in[1,4 /(2 \alpha+1)) \\
\ln (n)^{3 / 2} / n^{2} & q=4 /(2 \alpha+1) \\
\ln (n) / n^{2} & q \in(4 /(2 \alpha+1), 2 / \alpha] \\
1 / n^{\beta} & q>2 / \alpha, \beta \in(1,2) \text { arbitrary }\end{cases}
\end{aligned}
$$

holds.

Proof. This follows from

$$
\begin{aligned}
&\left\|\left(\mathcal{H} u\left(x_{j}^{(n, q)}\right)-\mathcal{H}^{(n, q)} u\left(x_{j}^{(n, q)}\right)\right)_{j}\right\|_{2, n q} \\
& \leq\left\|\left(\mathcal{H} u\left(x_{j}^{(n, q)}\right)-\left(\mathcal{H} P^{(n, q)} u\right)\left(x_{j}^{(n, q)}\right)\right)_{j}\right\|_{2, n, q} \\
& \quad+\left\|\left(\left(\mathcal{H} P^{(n, q)} u\right)\left(x_{j}^{(n, q)}\right)-\mathcal{H}^{(n, q)} u\left(x_{j}^{(n, q)}\right)\right)_{j}\right\|_{2, n, q} .
\end{aligned}
$$

Corollary 3.3. and Lemma 3.5.

4. Numerical results. First we calculate for the function

$$
u_{1}(x):= \begin{cases}(2(1+\cos (x)))^{1 / 4} \sin (x / 4) & x \in[0, \pi] \\ -(2(1+\cos (x)))^{1 / 4} \cos (x / 4) & x \in(\pi, 2 \pi]\end{cases}
$$

the approximation $\mathcal{H}^{(n, q)} u_{1}$. The function $\mathcal{H} u_{1}$ is known explicitly: 
TABLE $1 . u_{1}(\cdot)$.

\begin{tabular}{|l|c|c|c|c|}
\hline$q=1$ & err $_{\infty, n}$ & oc & $\operatorname{err}_{2, n, q}$ & oc \\
\hline$n=10$ & $3.9 \times 10^{-1}$ & & $3.1 \times 10^{-1}$ & \\
$n=21$ & $2.5 \times 10^{-1}$ & 0.58 & $1.4 \times 10^{-1}$ & 1.05 \\
$n=40$ & $1.8 \times 10^{-1}$ & 0.49 & $7.4 \times 10^{-2}$ & 1.03 \\
$n=81$ & $1.2 \times 10^{-1}$ & 0.55 & $3.6 \times 10^{-2}$ & 1.02 \\
$n=160$ & $9.1 \times 10^{-2}$ & 0.47 & $1.8 \times 10^{-2}$ & 1.00 \\
$n=321$ & $6.2 \times 10^{-2}$ & 0.54 & $9.0 \times 10^{-3}$ & 1.01 \\
\hline$q=2$ & \multicolumn{5}{|l|}{} \\
\hline$n=10$ & $1.9 \times 10^{-1}$ & & $7.4 \times 10^{-2}$ & \\
$n=21$ & $9.2 \times 10^{-2}$ & 0.97 & $2.0 \times 10^{-2}$ & 1.75 \\
$n=40$ & $4.6 \times 10^{-2}$ & 1.06 & $4.7 \times 10^{-3}$ & 2.20 \\
$n=81$ & $2.4 \times 10^{-2}$ & 0.96 & $1.4 \times 10^{-3}$ & 1.73 \\
$n=160$ & $1.2 \times 10^{-2}$ & 1.05 & $3.0 \times 10^{-4}$ & 2.25 \\
$n=321$ & $5.9 \times 10^{-3}$ & 0.96 & $9.1 \times 10^{-5}$ & 1.70 \\
\hline$q=4$ & \multicolumn{5}{|l|}{} \\
\hline$n=10$ & $1.1 \times 10^{-1}$ & \multicolumn{5}{|l|}{$1.2 \times 10^{-1}$} & \\
$n=21$ & $3.3 \times 10^{-2}$ & 1.56 & $2.4 \times 10^{-2}$ & 2.16 \\
$n=40$ & $1.0 \times 10^{-2}$ & 1.83 & $6.2 \times 10^{-3}$ & 2.08 \\
$n=81$ & $3.1 \times 10^{-3}$ & 1.69 & $1.5 \times 10^{-3}$ & 2.05 \\
$n=160$ & $8.6 \times 10^{-4}$ & 1.88 & $3.7 \times 10^{-4}$ & 2.01 \\
$n=321$ & $2.5 \times 10^{-4}$ & 1.81 & $1.0 \times 10^{-4}$ & 1.89 \\
\hline$q=5$ & \multicolumn{5}{|l}{} \\
\hline$n=10$ & $1.1 \times 10^{-1}$ & \multicolumn{5}{|l}{$1.8 \times 10^{-1}$} & \\
$n=21$ & $3.1 \times 10^{-2}$ & 1.76 & $3.6 \times 10^{-2}$ & 2.17 \\
$n=40$ & $9.0 \times 10^{-3}$ & 1.90 & $9.2 \times 10^{-3}$ & 2.10 \\
$n=81$ & $2.4 \times 10^{-3}$ & 1.88 & $2.2 \times 10^{-3}$ & 2.06 \\
$n=160$ & $6.3 \times 10^{-4}$ & 1.96 & $5.5 \times 10^{-4}$ & 2.03 \\
$n=321$ & $1.5 \times 10^{-4}$ & 2.02 & $1.4 \times 10^{-4}$ & 1.93 \\
\hline
\end{tabular}




$$
\mathcal{H} u_{1}(x)=- \begin{cases}(2(1+\cos (x)))^{1 / 4} \cos (x / 4)-1 & x \in[0, \pi] \\ (2(1+\cos (x)))^{1 / 4} \sin (x / 4)-1 & x \in(\pi, 2 \pi]\end{cases}
$$

We have $u_{1} \in C_{\bullet}^{2,1 / 2}([0,2 \pi], \mathbf{R})$. The errors

$$
\left\|\left(\mathcal{H} u\left(x_{j}^{(n, q)}\right)-\left(\mathcal{H}^{(n, q)} u\right)\left(x_{j}^{(n, q)}\right)\right)_{j}\right\|_{\infty, n}
$$

and

$$
\left\|\left(\mathcal{H} u\left(x_{j}^{(n, q)}\right)-\left(\mathcal{H}^{(n, q)} u\right)\left(x_{j}^{(n, q)}\right)\right)_{j}\right\|_{2, n, q}
$$

are abbreviated by $\operatorname{err}_{\infty, n}$ and $\operatorname{err}_{2, n, q}$. The order of convergence (oc) is estimated by

$$
\ln \left(\frac{\operatorname{err}_{\infty, n_{1}}}{\operatorname{err}_{\infty, n_{2}}}\right) / \ln \left(\frac{n_{1}}{n_{2}}\right)
$$

and in exactly the same way for $\operatorname{err}_{2, n, q}$. We get the results in Table 1. The results show that, for the function $u_{1}, q>2$ has to be chosen to get the optimal order for the $\|\cdot\|_{2, n, q}$-norm and $q>4$ for the $\|\cdot\|_{\infty, n}$-norm.

As an application we approximate the solution of equation (8) on the interval $[-\pi, \pi]$ with the following data

$$
\begin{aligned}
v(x) & :=v_{1}(\gamma(x)), \quad x \in[-\pi, \pi], \\
v_{1}(x) & := \begin{cases}-\sin (x / 2)(\cos ((2 \pi+x) / 4), \sin ((2 \pi+x) / 4))^{T} & x \in[-\pi, 0], \\
\sin (x / 2)(\cos (x / 4), \sin (x / 4))^{T} & x \in[0, \pi],\end{cases} \\
\gamma(x) & := \begin{cases}-\pi(1-\cos (x / 2)) & x \in[-\pi, 0], \\
\pi(1-\cos (x / 2)) & x \in[0, \pi],\end{cases} \\
f & \equiv 1 .
\end{aligned}
$$

The curve $\Gamma$ is closed and smooth with the exception of $v(0)$, and the angle between $v_{+}^{\prime}(0)$ and $v_{-}^{\prime}(0)$ is $\pi / 2$. The solution $u_{2}(x)$ of this equation is in $C_{\bullet}^{2,1 / 3}([-\pi, \pi], \mathbf{R})$ (see [2, Section 5$]$ ). Therefore, we expect $q / 3$ or $q(1 / 2+1 / 3)$, respectively, as order of convergence-assuming stability, which I will study in a forthcoming paper. We compute the approximation $u^{(n, q)} \in \mathcal{P}^{(n, q)}$ as the solution of the following linear system

$$
\left(I-\mathcal{H}^{(n, q)} D(K-A)+J(K-A)\right) u^{(n, q)}=\left(-\mathcal{H}^{(n, q)} D+J\right) f .
$$


TABLE 2. $u_{2}(\cdot)$.

\begin{tabular}{|l|c|c|c|c|}
\hline$q=1.0$ & $\operatorname{err}_{\infty, n}$ & oc & $\operatorname{err}_{2, n, q}$ & oc \\
\hline$n=10$ & $2.4 \times 10^{-1}$ & & $1.9 \times 10^{-1}$ & \\
$n=20$ & $1.9 \times 10^{-1}$ & 0.33 & $1.1 \times 10^{-1}$ & 0.83 \\
$n=40$ & $1.5 \times 10^{-1}$ & 0.33 & $6.1 \times 10^{-2}$ & 0.83 \\
$n=80$ & $1.2 \times 10^{-1}$ & 0.33 & $3.4 \times 10^{-2}$ & 0.83 \\
$n=160$ & $9.6 \times 10^{-2}$ & 0.33 & $1.9 \times 10^{-2}$ & 0.83 \\
$n=320$ & $7.6 \times 10^{-2}$ & 0.33 & $1.1 \times 10^{-2}$ & 0.83 \\
\hline$q=2.4$ & \multicolumn{5}{|l|}{} \\
\hline$n=10$ & $1.4 \times 10^{-1}$ & & $4.0 \times 10^{-2}$ & \\
$n=20$ & $7.9 \times 10^{-2}$ & 0.80 & $1.0 \times 10^{-2}$ & 1.94 \\
$n=40$ & $4.5 \times 10^{-2}$ & 0.80 & $2.7 \times 10^{-3}$ & 1.94 \\
$n=80$ & $2.6 \times 10^{-2}$ & 0.80 & $7.0 \times 10^{-4}$ & 1.96 \\
$n=160$ & $1.5 \times 10^{-2}$ & 0.80 & $1.8 \times 10^{-4}$ & 1.98 \\
$n=320$ & $8.6 \times 10^{-3}$ & 0.80 & $4.4 \times 10^{-5}$ & 2.00 \\
\hline$q=4.0$ & \multicolumn{5}{|l|}{} \\
\hline$n=10$ & $7.7 \times 10^{-2}$ & \multicolumn{5}{|l|}{$5.6 \times 10^{-2}$} & \\
$n=20$ & $3.1 \times 10^{-2}$ & 1.31 & $1.3 \times 10^{-2}$ & 2.11 \\
$n=40$ & $1.2 \times 10^{-2}$ & 1.32 & $3.3 \times 10^{-3}$ & 1.95 \\
$n=80$ & $5.0 \times 10^{-3}$ & 1.33 & $8.5 \times 10^{-4}$ & 1.97 \\
$n=160$ & $2.0 \times 10^{-4}$ & 1.33 & $2.1 \times 10^{-4}$ & 2.04 \\
$n=320$ & $7.8 \times 10^{-4}$ & 1.33 & $4.5 \times 10^{-5}$ & 2.22 \\
\hline$q=6.0$ & \multicolumn{5}{|l}{} \\
\hline$n=10$ & $5.9 \times 10^{-2}$ & \multicolumn{5}{|l}{$1.1 \times 10^{-1}$} & \\
$n=20$ & $2.4 \times 10^{-2}$ & 1.30 & $2.8 \times 10^{-2}$ & 1.99 \\
$n=40$ & $6.3 \times 10^{-3}$ & 1.94 & $6.7 \times 10^{-3}$ & 2.03 \\
$n=80$ & $1.6 \times 10^{-3}$ & 2.01 & $1.8 \times 10^{-3}$ & 1.93 \\
$n=160$ & $3.8 \times 10^{-4}$ & 2.04 & $4.5 \times 10^{-4}$ & 1.98 \\
$n=320$ & $8.9 \times 10^{-5}$ & 2.09 & $1.1 \times 10^{-4}$ & 2.04 \\
\hline
\end{tabular}


Here the operator $J$ is defined by

$$
J u:=\frac{1}{2 \pi} \int_{-\pi}^{\pi} u(x) d x, \quad u \in C([-\pi, \pi]),
$$

and $D$ is the differentiation operator. The kernels of the integral operators $D(K-A)$ and $(K-A)$ are known, and we compute $D(K-$ $A) u^{(n, q)}$ and $(K-A) u^{(n, q)}$ with the midpoint rule. The numerical solution with $q=6$ and $n=1280$ is used as a reference solution for the results in Table 2 .

The numerical results show that our estimates in Theorems 3.4 and 3.6 describe the order of convergence rather exactly.

Acknowledgment. I thank Prof. Dr. C. Schneider for giving me the idea to write this paper and for many suggestions.

\section{REFERENCES}

1. G.A. Chandler, Midpoint collocation for Cauchy singular integral equations, Numer. Math. 62 (1992), 483-503.

2. J. Elschner and I.G. Graham, An optimal order collocation method for first kind boundary integral equations on polygons, Numer. Math. 70 (1995), 1-37.

3. A. Gerasoulis, The use of piecewise quadratic polynomials for the solution of singular integral equations of Cauchy type, Comp. Math. Appl. 8 (1982), 15-22.

4. A. Gerasoulis and R.P. Srivastav, A method for the numerical solution of singular integral equations with a principal value integral, Internat. J. Engrg. Sci. 19 (1981), 1293-1298.

5. S.G. Michlin and S. Prößdorf, Singuläre Integraloperatoren, Akademie-Verlag, Berlin, 1980.

6. N.I. Muskhelishvili, Singular integral equations, P. Noordhoff V.V., GroningenHolland, 1953.

7. J.R. Rice, On the degree of convergence of nonlinear spline approximation, in Approximation with special emphasis on spline functions (I.J. Schoenberg, ed.), Academic Press, New York, 1969.

8. L.J. Slater, Generalized hypergeometric functions, Cambridge University Press, Cambridge, 1966. 